\title{
TAMENESS IMPLIED BY EXTENDING A HOMEOMORPHISM TO A POINT
}

\author{
L. D. LOVELAND ${ }^{1}$
}

A 2-sphere is a homeomorphic image of the unit sphere $B$ defined in $E^{3}$ by the equation $x^{2}+y^{2}+z^{2}=1$. A 2 -sphere $S$ is defined to be tame in $E^{3}$ if there exists a homeomorphism of $S$ onto $B$ that can be extended to a homeomorphism of $E^{3}$ onto itself. Stated another way, $S$ is tame if some homeomorphism of $E^{3}-S$ onto $E^{3}-B$ exists that can be extended to take $S$ onto $B$. The main result in this note states that $S$ is tame if, for each point $p$ of $S$, a homeomorphism $h$ of $E^{3}-S$ onto $E^{3}-B$ exists such that $h$ extends to take $p$ onto some point $b \in B$. Actually such a homeomorphism need exist only for each point of a subset of $S$ whose complement in $S$ is a closed 0-dimensional set.

The above condition can also be used to characterize "one sided" tameness; that is, a crumpled cube $C$ is a 3-cell if and only if for each point $p$ of $\mathrm{Bd} C$ there exists a point $b \in B$ and a homeomorphism $h$ of Int $C \cup p$ onto Int $B \cup b$. In this case such a homeomorphism need exist only for the piercing points of $C$. Perhaps of interest in this respect are those crumpled cubes $C$ in $E^{3}$ having the property that $\mathrm{Bd} C$ is locally tame from Int $C$ modulo a 0 -dimensional set $W$ of points where $\mathrm{Bd} C$ is wild from Int $C$. Such an example has been described by Fox and Artin [7]. In case $W$ consists of a point $p$ it can be deduced from a result by Doyle and Hocking [6] that no homeomorphism exists between Int $C \cup q$ and Int $B \cup b$ as long as $q \neq p(b \in B)$. It follows from Theorem 2 that no such homeomorphism exists for any point of $\mathrm{Bd} C$.

We also show that $S$ is tame if for each $p$ in $S$ and for each $\epsilon>0$ there exist an $\epsilon$-disk $D$ on $S$ containing $p$ and a homeomorphism $h$ of $E^{3}-S$ onto $E^{3}-B$ such that $h$ extends to take the boundary of $D$ homeomorphically into $B$. For example this means that $S$ is tame if it has a curvilinear triangulation $T$ of arbitrarily small mesh such that some homeomorphism of $E^{3}-S$ onto $E^{3}-B$ can be extended to take the 1-skeleton of $T$ homeomorphically into $B$.

Doyle and Hocking [6] have defined a $K-R$ 3-manifold $M$ to be a 3-manifold-with-boundary such that Int $M$ and $\mathrm{Bd} M$ are topologically $E^{3}$ and $E^{2}$, respectively. Suppose that $M^{2}$ is a closed topo-

Presented to the Society, August 28, 1968 under the title Tameness implied by extensions of homeomorphisms; received by the editors August 1, 1968.

${ }^{1}$ This research was partially supported by NSF-GP-7058 and NSF-GP-8454. 
logical plane in a 3-manifold $M^{3}$ and $U$ is a component of $M^{3}-M^{2}$. It follows from Corollary 2 that $M^{2} \cup U$ is a $K-R$ manifold if, for each point $p \in M^{2}, U \cup p$ is topologically Int $B \cup b(b \in B)$. Furthermore, applying [6] we see that the $K-R$ manifold $M^{2} \cup U$ is topologically $E^{2} \times[0,1)$.

In the following definitions and in Lemma $1, V$ denotes a component of $E^{3}-S$ and $p$ denotes a point of $S$. A point set $V$ is called an open 3-cell if $V$ is homeomorphic to Int $B$. An $\epsilon$-set is a set having diameter less than the positive number $\epsilon$. A 2-sphere $S$ is said to be locally $\alpha$-capped in $V$ at $p$ if for each $\epsilon>0$ there exists an $\epsilon$-disk $R$ on $S$ such that $p \in$ Int $R$ and for each $\alpha>0$ there is an open $\epsilon$-disk $D$ in $V \cap N(S, \alpha)$ such that Bd $D$ lies in $S-R$ and $R$ lies on the boundary of an $\epsilon$-component of $V-D$. The following lemma is easily proved and appears as Theorem 1 of [10].

Lemma 1. If $S$ is locally $\alpha$-capped in $V$ at $p$, then $V$ is locally simply connected at $p$.

THEOREM 1. If $p$ is a point of $S$ and there exists a homeomorphism of Int $S \cup p$ onto Int $B \cup b(b \in B)$, then Int $S$ is locally simply connected at $p$.

Proof. We shall show that $S$ is locally $\alpha$-capped in Int $S$ at $p$ and then rely on Lemma 1 to complete the proof. Let $\epsilon>0$, let $U$ be an $\epsilon / 2$-neighborhood of $p$ restricted to Int $S \cup_{p}$, and let $h$ be a homeomorphism as given in the hypothesis. There is a disk $E$ in $B$ such that $h(p) \in$ Int $E \subset E \subset \operatorname{cl}(h(U))$ and $E \cap \operatorname{cl}($ Int $B-h(U))=\varnothing$ (otherwise some sequence $\left\{h\left(p_{i}\right)\right\}$ of points in Int $B-h(U)$ converges to $h(p)$ without $\left\{p_{i}\right\}$ converging to $\left.p\right)$. Let $I$ be the unit interval $[0,1]$. Since $B \cup$ Int $B$ is a 3-cell, there exists a homeomorphism $f$ of $E \times I$ into $B \cup$ Int $B$ such that $f(E \times 0)=E$ and $f(E \times(0,1)) \subset h(U)$. Let $A^{\prime}$ be the open annulus $f(\mathrm{Bd} E \times(0,1))$ and let $A=h^{-1}\left(A^{\prime}\right)$. Then $A \subset U$ and one component $M$ of $\mathrm{Bd} A$ lies in $S-p$. Select a disk $R$ such that $p \in$ Int $R \subset R \subset S-M$, and let $\alpha>0$. If $H$ is the set of all points of Int $S$ within $\alpha$ of $S$, then $h(H)$ contains $f(E \times(0, t))$ for some $t$ such that $0<t<1$. Let $D^{\prime}$ be the open disk $f(\operatorname{Bd} E \times(0, t)) \cup f(E \times t)$. Then $D=h^{-1}\left(D^{\prime}\right)$ is an open $\epsilon$-disk in $N(S, \alpha)$ such that $\operatorname{Bd} D=M C S-R$, Certainly $R$ lies on the boundary of an $\epsilon$-component of Int $S-D$, so $S$ is locally $\alpha$-capped in Int $S$ at $p$.

Corollary 1. Suppose $M$ is a compact connected 2-manifold that separates a connected 3-manifold $M^{3}, U$ is a component of $M^{3}-M$, and $K$ is a polyhedral homeomorphic image of $M$ in $M^{3}$. If there is a component $V$ of $M^{3}-K$ such that, for each $p \in M, a$ point $b \in K$ and $a$ homeomorphism taking $U \cup p$ onto $V \cup b$ exist, then $M$ is tame from $U$. 
Proof. The proof given for Theorem 1 is not only valid for 2spheres but will also hold for 2-manifolds as in the hypothesis. Thus $U$ is locally simply connected at each point of $M$, and it follows from [2] that $M$ is tame from $U$.

Remark. In case $M$ is a 2 -sphere Corollary 1 can be improved somewhat by weakening the hypothesis. We proceed toward an improved version, Theorem 3 to follow, by establishing Lemma 2 and Theorem 2 below. Corollary 2 seems of interest relative to $K-R$ manifolds as defined in [6].

Corollary 2. Suppose $M$ is a closed topological plane in a 3-manifold $M^{3}$ and $U$ is a component of $M^{3}-M$. If, for each $p \in M$, a homeomorphism exists taking $U \cup p$ onto Int $B \cup b(b \in B)$, then $M \cup U$ is homeomorphic to $E^{2} \times[0,1)$.

Proof. As shown in the proof of Theorem $1 U$ is locally simply connected at each $p \in M$. Thus $M \cup U$ is a 3-manifold with boundary [2]. Now it follows from the corollary in [6] that the $K-R$ manifold $M \cup U$ is topologically $E^{2} \times[0,1)$.

Lemma 2. Suppose a 2-sphere $S$ in $E^{3}$ is locally tame from Int $S$ modulo a 0-dimensional set $W$ and some point $q$ in $S-W$ has the following property: There exists an arc $A$ and a sequence $\left\{S_{i}\right\}$ of 2-spheres converging to $S$ such that, for each $i, S_{i} \subset$ Int $S, S_{i} \cap A$ is a point at which $A$ pierces $S_{i}, q$ is an endpoint of $A$, and $S \cap A=\{q\}$. Then $S$ is tame from Int $S$.

Proof. Since Int $S$ is an open 3-cell, $S$ is locally tame from Int $S$ modulo one point $p$ [5]. We shall show that $S$ can be locally spanned from Int $S$ at $p$ and then apply the results of [4] to conclude that $S$ is tame from Int $S$. There is some resemblance between our proof and the proof given for Theorem 1 in [5].

Let $\epsilon>0$ and choose $\delta$ such that $0<7 \delta<\epsilon$ and $\delta<d(p, A)$. There exists a disk $R$, a locally polyhedral annulus $B$, and a simple closed curve $J$ such that $p \in$ Int $R \subset S$, Bd $R \subset B, B-B d R \subset$ Int $S$, Diam $(B \cup R)<\delta, J \cap S=\{p, q\}, J \cap B=\varnothing, A \subset J$, and $J$ pierces $S$ at both $p$ and $q$. Let $J_{1}=\mathrm{Bd} B-\mathrm{Bd} R$. There is a 3-cell $K$ such that $J_{1} \subset$ Int $K \subset K \subset$ Int $S$, there is an annulus $B^{\prime} \subset B$ such that $\mathrm{Bd} R$ $\subset \mathrm{Bd} B^{\prime}$ and $B^{\prime} \cap K=\varnothing$, and there is a subarc $F$ of $J$ such that $p \in \mathrm{Bd} F, F-p \subset$ Int $S, F \cap K=\varnothing$, and Diam $F<\delta$. Using the hypothesis, we choose a polyhedral 2 -sphere $S^{\prime}$ in Int $S$ such that $S^{\prime} \cap(J-A) \subset F, K \subset$ Int $S^{\prime}, S^{\prime} \cap B \subset B^{\prime}, S^{\prime} \cap A$ is a point,

(1) $S^{\prime} \subset N(S, \delta)$, and

(2) $d(x, S)<d(K, S)$ for all $x \in S^{\prime}$. 
We also assume that $S^{\prime} \cap B^{\prime}$ consists of a finite collection of disjoint simple closed curves $L_{1}, L_{2}, \cdots, L_{n}$ no one of which can be contracted in $B$. Then each $L_{i}$ bounds a disk $E_{i}$ on $S^{\prime}$ where $E_{\boldsymbol{i}} \cap A=\varnothing$. We choose an integer $k$ such that Int $E_{k}$ contains no $L_{i}$. Then $L_{k}$ and Bd $R$ bound an annulus $B^{\prime \prime}$ on $B^{\prime}$ such that $B^{\prime \prime} \cup E_{k}$ is a disk $D$ lying, except for its boundary $\mathrm{Bd} R$, in Int $S$. Notice that $D \cap(K \cup A)=\varnothing$. In the next paragraph we complete the proof by showing diam $D<\epsilon$.

Let $E_{k}=E$. Since $J$ links Bd $E$ there exists a point $a \in J \cap I n t E$. From the construction we see that $a \in F$, so $d(p, a)<\delta$. Suppose Diam $E$ is greater than $6 \delta$. Then some point $b \in \operatorname{Int} E$ exists such that $d(a, b)>3 \delta$ and there is an arc $a b$ from $a$ to $b$ in Int $E$. It follows from (1) and (2) that there exists a point $c \in S$ such that the segment $b c$ does not intersect $K \cup B \cup R$ and $d(, b c)<\delta$. The union of a subarc of $F$ from $p$ to $a, a b, b c$, and an open arc in Ext $S$ from $c$ to $p$ is a simple closed curve $J^{\prime}$ such that $J^{\prime} \cap B=\varnothing, J^{\prime} \subset E^{3}-K$, and $J^{\prime}$ links $\mathrm{Bd} R$. This means that $J^{\prime}$ links $J_{1}[1]$; however, this is a contradiction since $J_{1}$ can be contracted in the 3 -cell $K$. Hence Diam $E \leqq 6 \delta$. Since Diam $B^{\prime \prime}<\delta$, we know that Diam $D<7 \delta<\epsilon$.

THEOREM 2. Suppose that $S$ is a 2-sphere in $E^{3}$ such that the set $W$ of points where $S$ is wild from Int $S$ is 0-dimensional. If for some point $q \in S$ there exists a homeomorphism h of Int $S \cup q$ onto Int $B \cup b(b \in B)$, then $S \cup \operatorname{Int} S$ is a 3-cell.

Proof. It is easy to find an $\operatorname{arc} A^{\prime}$ and a sequence $\left\{S_{i}{ }^{\prime}\right\}$ of round 2 -spheres in the range of $h$ such that their inverse images under $h$ satisfy the hypothesis of Lemma 2. If $q \in S-W$, it follows from Lemma 2 that $S$ is tame from Int $S$.

If $q \in W$ then $S$ is locally tame from Int $S$ at each point of $S-q$ [5]. That $S$ is locally tame from Int $S$ in this case follows from Theorem 1 and [2].

Theorem 3. If there exists a closed 0-dimensional subset $G$ of a 2sphere $S$ such that, for each point $p \in S-G$, there exists a homeomorphism of $p \cup \operatorname{Int} S$ onto $b \cup \operatorname{Int} B$, then $S \cup \operatorname{Int} S$ is a 3-cell.

Proof. Since $S-G$ is open it follows from Theorem 1 and [2] that $S$ is locally tame from Int $S$ at each point of $S-G$. Thus Theorem 3 follows from Theorem 2 .

A point $p$ of a 2 -sphere $S$ has been called a piercing point of the crumpled cube $C=S \cup$ Int $S$ if and only if there is a homeomorphism $h$ of $C$ into $E^{3}$ such that $h(S)$ can be pierced at $h(p)$ by a tame arc [13]. 
CoROllaRy 3. If $S$ is a 2-sphere in $E^{3}$ such that, for each piercing point $p$ of $S \cup \operatorname{Int} S$, there exists a homeomorphism of Int $S \cup p$ onto Int $B \cup b(b \in B)$, then $S \cup$ Int $S$ is a 3-cell.

Proof. From the fact that $S \cup \operatorname{Int} S$ has piercing points [13] and the hypothesis, we see that Int $S$ is an open 3-cell. Then $S \cup \operatorname{Int} S$ has at most one nonpiercing point (see [11] or [14]), so Corollary 3 follows from Theorem 3 .

THEOREM 4. If there is a closed 0-dimensional subset $G$ of a 2-sphere $S$ such that for each $p \in S-G$ there exists a homeomorphism of $\left(E^{3}-S\right) \cup p$ onto $\left(E^{3}-B\right) \cup b$, then $S$ is tame.

Proof. We may think of $S$ as lying in $S^{3}$ so that it follows from Theorem 3 that $S$ bounds two 3 -cells in $S^{3}$. Thus $S$ is tame.

Corollary 4. If $S$ is a 2-sphere in $E^{3}$ such that, for each point $p$ of $S$ that lies in a tame arc on $S$, there exists a homeomorphism of $\left(E^{3}-S\right)$ $\cup P$ onto $\left(E^{3}-B\right) \cup b$, then $S$ is tame.

Proof. Corollary 4 follows from Theorem 4 because at most two points of $S$ fail to lie in tame arcs in $S$ (see [8] and [11] or [14]).

TheOREM 5. Let $S$ be a 2-sphere in $E^{3}$ and let $\epsilon>0$. If for each $p \in S$ there exist an $\epsilon$-disk $D$ in $S$ containing $p$ and a homeomorphism $h$ of Int $S$ onto Int $B$ such that $h$ extends to take $\mathrm{Bd} D$ homeomorphically into $B$, then $S \cup$ Int $S$ is a 3-cell.

Proof. Let $p \in S$ and notice that Int $S$ is locally simply connected at $p$ if $p$ lies in the boundary of some disk $D$ as given in the hypothesis (see Theorem 1). Otherwise $p$ lies in the interiors of arbitrarily small such disks, and we are able to prove that $S$ is locally $\alpha$-capped from Int $S$ at $p$. Once this is done we rely on Lemma 1 and [2] to complete the proof.

Let $U$ be an open set containing $p$, let $D$ be a disk such that $p \in$ Int $D \subset D \subset U \cap S$, and let $h$ be a homeomorphism of Int $S$ onto Int $B$ that extends to take $\mathrm{Bd} D$ homeomorphically into $B$. As in the proof of Theorem 1, we choose a disk $E^{\prime}$ in $h(U \cap$ Int $S) \cup h(\operatorname{Bd} D)$ such that $\mathrm{Bd} E^{\prime}=h(\mathrm{Bd} D)$, and we let $E=h^{-1}\left(E^{\prime}\right)$. Such a disk $E$ can be chosen in any neighborhood of $S$, so $S$ is locally $\alpha$-capped from Int $S$ at $p$.

THEOREM 6. If for each point $p$ of a closed subset $F$ of a 2-sphere $S$ in $E^{3}$ there exists a homeomorphism $h$ of Int $S \cup p$ onto Int $B \cup b$, then $F$ lies on the boundary of a 3-cell. 
Proof. From Theorem 1 we see that Int $S$ is locally simply connected at each point of $F$. Now the techniques in the proof of Theorem 1 in [2] can be applied, as indicated in the proof of Theorem 10 of [9], to yield Property ( ${ }^{*}, F$, Int $S$ ); that is, $S$ can be side approximated from Int $S$ missing $F$ (see [8] or [9] for a precise definition). The reason no restriction is placed on the size of the components of $F$ in Theorem 6 is that the local simple connectedness of Int $S$ at each point of $F$ is a much stronger hypothesis than that of Theorem 10 of [9]. We omit the tedious details since they are like those of [9]. Once $(*, F$, Int $S)$ is known the conclusion of Theorem 6 follows from [12].

THEOREM 7. If $S$ is a 2-sphere in $E^{3}$ and $p$ is a point of $S$ such that there exists a homeomorphism of Int $S \cup p$ onto Int $B \cup b(b \in B)$, then $p$ is a piercing point of $S \cup \operatorname{Int} S$.

Proof. As indicated in the proof of Theorem 6, Property (*, $p$, Int $S$ ) can be established. This is sufficient for $p$ to be a piercing point of $S \cup \operatorname{Int} S[11]$.

REMARK. Of course the converse to Theorem 7 is false even if Int $S$ is an open 3 -cell (see Theorem 2 and an appropriate example in [7]).

Theorem 8. Suppose $F$ is a set as given in Theorem 6. If for each point $p$ of $F$ there exists a homeomorphism of $\left(E^{3}-S\right) \cup p$ onto $\left(E^{3}-B\right)$ $\cup b(b \in B)$, then $F$ lies on a tame 2-sphere.

Proof. The proof here is much the same as that outlined for Theorem 6. We first obtain Property $(*, F, S)$, then we appeal to Theorem 6 of [9].

\section{REFERENCES}

1. R. H. Bing, Approximating surfaces with polyhedral ones, Ann. of Math. (2) 65 (1957), 456-483.

2. - A surface is tame if its complement is 1-ULC, Trans. Amer. Math. Soc. 101 (1961), 294-305.

3. - Each disk in $E^{3}$ contains a tame arc, Amer. J. Math. 84 (1962), 583-590.

4. C. E. Burgess, Characterizations of tame surfaces in $E^{3}$, Trans. Amer. Math. Soc. 114 (1965), 80-97.

5. - Criteria for a 2-sphere in $S^{3}$ to be tame modulo two points, Michigan Math. J. 14 (1967), 321-330.

6. P. H. Doyle and J. G. Hocking, Special n-manifolds with boundary, Proc. Amer. Math. Soc. 16 (1965), 133-135.

7. R. H. Fox and E. Artin, Some wild cells and spheres in three-dimension space, Ann of Math. (2) 49 (1948), 979-990. 
8. D. S. Gillman, Side approximation, missing an arc, Amer. J. Math. 85 (1963), 459-476.

9. L. D. Loveland, Tame subsets of spheres in $E^{3}$, Pacific J. Math. 19 (1966), 489517.

10. - Conditions implying that a 2-sphere is almost tame, Trans. Amer. Math. Soc. 131 (1968), 170-181.

11. - Piercing points of crumpled cubes, Trans. Amer. Math. Soc. 143 (1969), 145-152.

12. - Sufficient conditions for a closed set to lie on the boundary of a 3-cell, Proc. Amer. Math. Soc. 19 (1968), 649-652.

13. Joseph Martin, The sum of two crumpled cubes, Michigan Math. J. 13(1966), 147-151.

14. D. R. McMillan, Jr., Piercing a disk along a cellular set, Proc. Amer. Math. Soc. 19 (1968), 153-157.

Utah State University aNd

UNIVERSITY OF UTAH 